\title{
The Correlation between Sleep Deprivation and Motor Skill among Night Shift Residents in DR. M.Djamil Hospital, Padang
}

\author{
Hendra Permana ${ }^{1,2}$, Yaumi Faiza ${ }^{1,3}$ \\ \{saint_check_lie@yahoo.com\} \\ Neurology Department, Faculty of Medicine, Andalas University / DR. M.Djamil Hospital, Padang, \\ Indonesia ${ }^{1,3}$ \\ Sleep Disorder Study Group, Indonesian Neurological Association, Padang, Indonesia ${ }^{2}$
}

\begin{abstract}
Sleep deprivation is common among residents. It contributes to daytime sleepiness and exhaustion that causing deterioration of productivity. This condition may affect their motor performance. As a consequence, sleep deprivation can increase the risk of human error among physician. This study aimed to evaluate the correlation between sleep deprivation and motor skill in night shift residents. We conducted a cross-sectional study in DR. M. Djamil hospital, Padang between July to August 2016. A total of 51 residents were recruited, related to night shift one day before the study. Those with chronic illness, consuming sedative drugs, and visual impairment was excluded. The Epworth Sleepiness Scale (ESS) was used to examine the duration of sleep in sleep-deprived subjects, and motor skills were evaluated using the Purdue Pegboard Test. The relationship between variables was analyzed using Kendall's Tau b correlation, and p-value $<0.05$ was considered as statistically significant. The mean of sleep duration was $2.37 \pm 0.894$ hours. The mean of Purdue Pegboard Test was 20.14 seconds on the right, 18.63 seconds on the left, and 15.61 seconds on both hands respectively. There was no correlation between sleep deprivation and motor performance of right hand $(\mathrm{p}=0.737)$, left hand $(\mathrm{p}=0.541)$, nor in both hands $(\mathrm{p}=0.400)$. There was a significant correlation between sleep deprivation and motor performance of both hands in night shift residents.
\end{abstract}

Keywords: Motor Performance, Night Shift Resident, Sleep Deprivation.

\section{Introduction}

The World Health Organization report on 2004 stated that primary insomnia has the deleterious effect on a healthy living, which 3,6 million years of productive life is lost worldwide [1]. Sleep disorder and sleep deprivation are often found in modern society today [2]. Many professions are commonly related to long hours and overnight working [3]. The effects of sleep deprivation and sleep disorder are common in industrialized countries, such as airlines, longdistance expedition services, factories with night shift workers and emergency units $[4,5]$. However, it is estimated that the effect of sleep deprivation in a wider scope is far greater. Most studies have shown that the population is increasingly exposed to sleep deprivation and shows a picture of a constant decrease in sleep quality. Some researchers concluded that nearly $20 \%$ of sleep deprivation cases were found in adults [6]. These disorders are related to changes in environmental and social conditions, such as social activities that are not related to changes in 
time, night shift and 24-hour work activity or more. This impacts on health conditions and chronic diseases that will more increase as the increasing of sleep disorder in society. [7].

Most adults need 7-8 hours a day to sleep based on a recommendation from National Sleep Foundation [8,9]. The regulation of sleep duration is influenced by two factors like genetic and environmental factors. Genetic factors have an important role in determining the duration of sleep needed and affect a person's ability to reduce the desired sleep duration. Environmental factors have varying effects on a person's duration and sleep patterns [10].

Sleep deprivation can affect motor skills. There is evidence from some studies that show the occurrence of impaired motor performance due to sleep deprivation. The effects of sleep deprivation on work that requires dexterity are reported compared to the effects of alcohol intoxication. In one study, motor performance on the person who remained awake for 24 hours was comparable to a person with 0.1 percent alcohol blood level [11,12,3]. Besides, Weinger et al. (2002) concluded the results of two meta-analysis studies from Koslowski et al. (1992) and Pilcher et al. (1996) that there were decreases in performance due to lack of sleep by 1.4 standard deviations compared to control group [13].

A resident who has a night shift has big potential to have sleep deprivation. This is maybe not a serious problem, but it can increase the risk of doing the doctor's professional duties. The level of human error could increase and can endanger patient safety, as well as the safety of the doctor itself. Resident as the foremost medical service in the teaching hospital and networking hospital are required to always be in good condition, even though the public and medical education recognize that long duration of work has contra productive effects. Long work duration causes a reduction on sleep time, disruption of the learning process and also increases the risk of medical errors. This condition is difficult to fix because some medical circles believe that the long duration of work is important to create a high standard of residency entrance. This condition is also used to reduce health facilities outlay [3].

Some findings show that there is a relationship between lack of sleep and various disorders of night shift residents. Although most of the study concluded that sleep deprivation was related to cognitive performance, mood, depression, but the impact to motor skill resulted in a contradictive manner. Some studies in surgical residents did not uniformly state that lack of sleep may cause errors in operative skills [14].

Our goals in this study were to quantify the sleep hour of night shift residents, assessing motor performance, and analyze the relationship between them.

\section{Materials and Method}

We conducted an observational study with cross-sectional design. This study was held for 2 months, starting from June 2016 to August 2016 at Dr. M. Djamil Hospital. A total of 35night shift residents were recruited in this study. They have been on night shift with sleep duration less than 4 hours. Subjects who had a chronic disease such as liver, kidney, lung disease, or visual impairments, taking tranquilizers and caffeine were excluded from this study. Sleep deprivation was assessed using the Epworth Sleepiness Scale (ESS). Motor skills were assessed by using the Purdue Pegboard test. The assessment was held on 0-6 hours after a 24hour shift.

Data were analyzed with the Statistical Program for Social Sciences (SPSS) software. Additional variables such as demographic data (age, sex, department, dominant hand) were also 
analyzed. The relationship between categorical variables was analyzed by Spearman analysis. The value of $\mathrm{p}<0.05$ was considered as statistically significant.

\section{Result}

All residents have completed the study as the written protocol. Table 1 showed the characteristics of subjects. The mean age of participants was 28.39 years old, which the youngest one was 22 years old and the eldest was 37 years old. Male resident was more dominant to join this study (51\%), and most of them came from the internal medicine department $(27.4 \%)$. All of the subjects were right-handed.

Table 1. Characteristics of Subjects

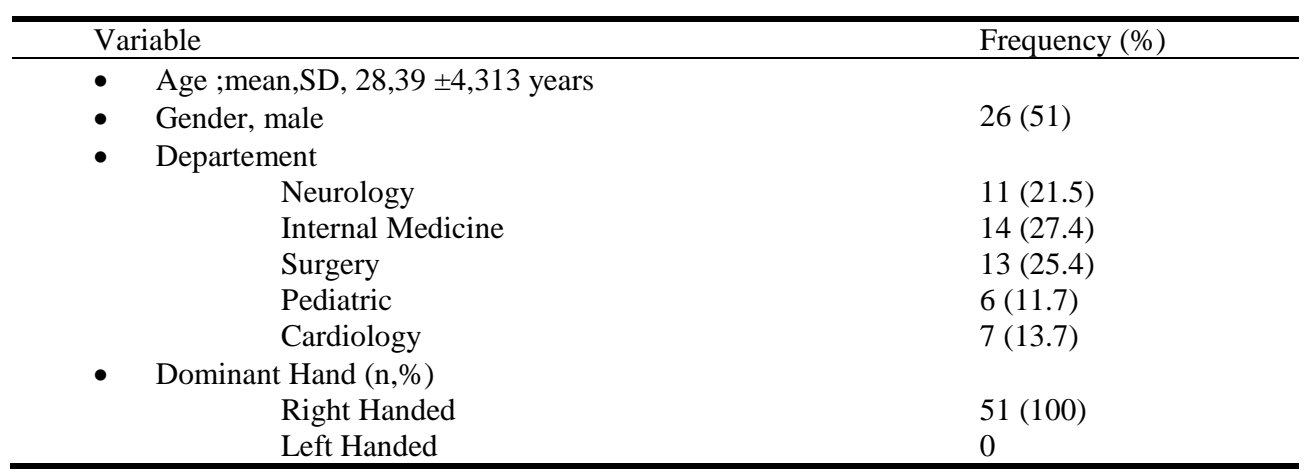

Sleep deprivation has been achieved for every resident, with a mean duration of sleep was 2.37 hours. Most residents have 3 hours of duration of sleep during this study. Increasingly, almost 20 percent of subjects were having a sleep for only 1 hour.

Based on the evaluation of motor skill after a night shift, the value of Purdue Pegboard Test showed between the normal range. The mean value was $20.14,18.63$, and 15.61 for the right hand, left hand and both hands respectively.

Table 2. Sleep duration and motor skills

\begin{tabular}{ll}
\hline Variables & $\mathrm{n}(\%)$ \\
\hline$-\quad$ Sleep duration (hours) & \\
1 & $10(19.6)$ \\
2 & $17(33.3)$ \\
3 & $20(39.2)$ \\
4 & $4(7.8)$ \\
Purdue Pegboard Test & \\
Right Hand, mean & 20.14 \\
Left Hand, mean & 18.63 \\
Both Hand, mean & 15.61 \\
\hline
\end{tabular}

We analyzed the relationship between sleep deprivation according to duration of sleep during the night shift and motor skill based on Purdue Pegboard Test values. The results were shown 
in table 3. From the analysis, we did not find any relationship between lack of sleep and motor skills, even with one hand or both hands.

Table 3. The Relationship between Sleep Deprivation and Motor Skill

\begin{tabular}{lll}
\hline Variables & $R$ & $p$-value \\
\hline Sleep deprivation * Right hand motor skills & .039 & .737 \\
Sleep deprivation * Left hand motor skills & -.070 & .541 \\
Sleep deprivation * Both hands motor skills & -.098 & .400 \\
\hline
\end{tabular}

Analyzed with Kendall's Tau b

\section{Discussion}

Physician ability to make better care may be affected by sleep disruption [13]. Since the death of Libby Zion in 1984 because of heavy loaded work hours, there is great concern about the regulation of work hours. As the law in 1989 issued in New York state, as well as the Accreditation Council for Graduate Medical Education (ACGME), some regulations to limit work hours in resident have been achieved. Naturally, the idea was to optimize patient care and outcomes, but the real effects of this were still on debate [14].

Many studies have proven the relationship between sleep deprivation and physical health. It may cause a decline in cognitive function [4]. The risk for academic failure in college student $[15,16]$, and also fatigue and weakness [17]. Hence, there are still some controversial findings of the relationship between sleep deprivation and motor performance, especially in night shift resident [13]. Several studies found that there is an inverse relationship between sleep deprivation with motor performance in on-call resident. Smith-Coggins et al. (1997) carried out a double-blind test of control placebo of the attending physician in the emergency department found that motor performance and mood became disturbed after a night shift in compared the motor performance and mood after day shift[18]. However, from the other study found that there is no relationship between motor skill nor cognitive impairment in resident after having the night shift. Reznick \& Folse (1987) evaluated the performance of 20 residents in the surgical training program [19]. They found that following sleep deprivation, most surgical errors were occurred but most of it just light and were not significant errors. Reimann et al. (2009) find that there is no relationship between sleep deprivation with cognitive performance of the neurology resident, they conclude that sleep deprivation might disturb in the longer and irregular working schedule, but not in the short period working schedule [20].

In our study, we found no correlation between sleep deprivation and motor skill of the night shift residents, even more than half of them were having a sleep below 3 hours during the oncall duty. These findings are quite interesting because we also include resident who needs more motor skill like surgical or cardiology resident. This finding is by the previous result which found that sleep deprivation in the shorter period did not impact the motor performance. However, these finding still need further research about another factor that might affect this relationship. Factors such as daytime sleep before shift might affect that relationship, and also cigarette consumption before night shift that might have a stimulant effect which can improve motor performance. 


\section{Conclusion}

There was no relationship between sleep deprivation and cognitive performance in night shift residents.

\section{References}

[1] Babson KA, Trainor CD, Feldner MT, Blumenthal H. 2010. A test of the effects of acute sleep deprivation on general and specific self-reported anxiety and depressive symptoms: an experimental extension. J Behav Ther Exp Psychiatry;41(3):297-303

[2] Tufik S, Andersen ML, Bittencourt LRA, De Mello MT. 2009. Paradoxical sleep deprivation: neurochemical, hormonal and behavioral alterations. Evidence from 30 years of research. An Acad Bras Cienc;81(3):521-538

[3] Philibert I. 2005. Sleep loss and performance in residents and nonphysicians: a meta-analytic examination. Sleep;28(11):1392-1402

[4] Alhola P, Polo-Kantola P. 2007. Sleep deprivation: impact on cognitive performance. Neuropsychiatric Disease and Treatment;3(5):553-567

[5] Dickinson DL, Drummond SPA. 2008. The effects of total sleep deprivation on bayesian updating. Judgment and Decision Making;3(2):181-190

[6] Roberts RE, Roberts CRR, Duong HT. 2009. Sleepless in adolescence:prospective data on sleep deprivation, health and functioning. J Adolesc;32(5):1045-1057

[7] Cappuccio FP, D'Elia L, Strazzulo P, Miller MA. 2010. Sleep duration and all-cause mortality: a systematic review and meta-analysis of prospective studies. Sleep;33(5):585-592

[8] Markov D. 2006. Normal sleep and circadian rhythms: neurobiologic and mechanisms underlying sleep and wakefulness. Psychiatric Clinics of North Americans;29(4):841-853

[9] Hirshkowitz M, Whiton K, Albert SM, Alessi C, Bruni O, DonCarlos L, et al. 2015. National sleep foundation's sleep time duration recommendations: methodology and results summary. Sleep Health; $1: 40-43$

[10] Sehgal A, Mignot E. 2011. Genetics of sleep and sleep disorders. Cell;146(2):194-207

[11] Williamson AM, Feyer AM. 2000. Moderate sleep deprivation produces impairments in cognitive and motor performance equivalent to legally prescribed level of alcohol intoxication. Occup Environ Med;57:649-655

[12] Baldwin DC, Daugherty SR. 2004. Sleep deprivation and fatigue in residency training: results of a national

[13] Weinger MB, Ancoli-Israel S. 2002. Sleep deprivationand clinical performance. JAMA;287(8):955-957.

[14] Ayalon, Frieddman F. 2006. The effect of sleep deprivation on fine motor coordination in obstetric and gynecology residents. Am J of Obstet Gynecol;199:576.e1-576.e5

[15] Gaultney JF. 2010. The prevalence of sleep disorders in college students: impact on academic performance. Journal of American College Health;59(2):91-97

[16] Amin HS, Almazroua IS, Alsahlan AS, Alrishan MA, Elmourad HM, Alotaibi MM, et al. 2015. Effect of sleep deprivation on the attitude and performance of medical students, Riyadh, Saudi Arabia. International Journal of Medical Science and Public Health:5(3);575-80

[17] Drake CL, Roehrs T, Richardson G, Walsh JK, Roth T. 2004.Shift work sleep disorder: prevalence and consequences beyond that of symptomatic day workers. Sleep;27(8):1453-62

[18] Smith-Coggins R, Rosekind M, Buccino et al. 1997. Rotating shiftwork schedules: can we enhance physician adaptation to night shifts? Acad Emerg Med,352:1191

[19] Reznick RK, Folse JR. 1987. The effects of sleep deprivation on performance of surgical residents. Am J Surg;154:520-525

[20] Reimann M, Manz R, Reichmann H, Ziemssen T. 2009. Education research: cognitive performance is preserved in sleep-deprived neurology residents. Neurology;73:e99-e103 